Horizons philosophiques

\title{
Montesquieu : questionnements du patriarcat ou prémices d'une restructuration du sexuel?
}

\section{Ilhame El Himdy}

Volume 6, numéro 2, printemps 1996

La philosophie sur Internet

URI : https://id.erudit.org/iderudit/801017ar

DOI : https://doi.org/10.7202/801017ar

Aller au sommaire du numéro

Éditeur(s)

Collège Édouard-Montpetit

ISSN

1181-9227 (imprimé)

1920-2954 (numérique)

Découvrir la revue

Citer cet article

El Himdy, I. (1996). Montesquieu : questionnements du patriarcat ou prémices d'une restructuration du sexuel? Horizons philosophiques, 6(2), 119-129.

https://doi.org/10.7202/801017ar d'utilisation que vous pouvez consulter en ligne.

https://apropos.erudit.org/fr/usagers/politique-dutilisation/ 


\section{MONTESQUIEU : \\ QUESTIONNEMENTS DU \\ PATRIARCAT OU PRÉMICES \\ D'UNE RESTRUCTURATION DU \\ SEXUEL?'}

Dans les Lettres persanes, la réflexion politique de Montesquieu s'accompagne d'une réflexion sur la condition des femmes ${ }^{2}$ et leur place dans la société. Les Lettres sont le récit d'une révolte, celle des femmes d'un harem oriental, contre le despotisme domestique d'un seigneurpersan, Usbeck. Pourraiton lire dans cette révolte une mise en question du système patriarcal? Les travaux de Pauline Kra et de Jeannette Geffrio Rosso vont dans ce sens. La première voit dans les Lettres un manifeste féministe qui montre la voie de la libération et la seconde considère le roman du sérail comme une condamnation de la domination de la femme par l'homme, condamnation basée sur un faux prétexte de la vertu. Vertu prenant ici le sens de fidélité conjugale révèle un désir de contrôler la sexualité féminine qui est à l'origine de tous les désordres.

Essayons pour notre part de voir plutôt dans les Lettres une démarche - identique à celle de L'esprit des lois - qui tend à scruter le système patriarcal dans le but d'en comprendre le mécanisme, d'en révéler les fondements, mais aussi d'en souligner les faiblesses. Nous partons cependant de l'hypothèse que dans ce récit d'une révolte contre le despotisme domestique, Montesquieu nous donne à voir ce qui pourrait se passer si les femmes sont trop libres ou trop réprimées. Le défi en somme serait de touver leur vraie place. Le va-et-vient entre lspahan et Paris suggère que la France aussi a besoin d'un régime domestique repensé pour mettre fin à une mobilité sociale incontrôlée

1. Communication présentée à Harvard, le 19 octobre 1995.

2. Montesquieu s'est intéressé aux différents facteurs qui affectent la condition féminine et déterminentl le caractère et le comportement des femmes. Voirà ce propos Del'esprit des lois, livres VII, XVI, XIX, XXIII, XXIV, ainsi que les Pensées. Les références auX textes de Montesquieu renvoient aux CEuvres complètes de Montesquieu dans la Pléiade, Paris : Gallimard, 1949. 
et au dangereux déclin de la population et de la prospérité.

Si l'introduction de deux orientaux dans la société européenne des Lettres persanes a permis de souligner le contraste entre les mœurs orientales et occidentales, entre l'asservissement des persanes et la relative liberté des femmes européennes, elle a surtout permis par son effet boomerang de décrire les formes universelles de la subordination des femmes ${ }^{3}$ et d'interroger les fondements de l'autorité patriarcale.

Les personnages d'Usbeck et de Rica véhiculent les idées de Montesquieu. À travers Rica, il évoque les possibilités d'une égalité naturelle des femmes et d'une identité des besoins masculins et féminins, besoins que réprime l'éducation. Avec le roman du sérail et à travers la relation d'Usbeck et ses femmes, il dénonce la pluralité des femmes et leur claustration, suggérant des similarités dérangeantes entre le traitement oppressif des femmes en Orient et celui soi-disant "éclairé» et différent des Européens. Enfin, nous verrons comment à travers certaines de ses réflexions, Usbeck lui-même questionne certains aspects du système despotique - domestique et politique - qui nuisent à l'État.

En scrutant les relations hommes /femmes, Montesquieu souligne que les fondements du système patriarcal contrairement à ce qui était communément admis - ne sont conformes ni à la Nature ni à la Raison. La subordination des unes aux autres n'ayant rien de naturel et n'étant en rien conforme à la raison, les principes du patriarcat sont présentés comme une construction fragile dont la structure est constamment en péril.

Le débat sur la nature et le traitement des femmes permet de souligner ce qui détermine les rapports entre les sexes, à savoir l'injustice dont le ressort n'est autre que l'éducation. La lettre XXXVIII écrite par Rica reprend ce débat en présentant différentes opinions masculines surla subordination des femmes

3. Tout comme les femmes orientales, les femmes européennes sont condamnées à être le jouet des hommes (voir à ce propos les lettres XLVIII et LXXVIII) et les victimes d'un système qui ne valorise que leur apparence (voir lettres LII, LV ET LIX). 
auxhommes. Dans la polémique entre Orientaux et Occidentaux sur l'autorité «naturelle» des hommes sur les femmes et le degré de liberté qui devraitêtre accordé à celles-ci, Montesquieu évoque d'abord les traditionnelles angoisses du patriarcat pour lequel la libération des femmes encouragerait l'absence de vertu et déstabiliserait l'autorité masculine. Puis, il ajoute une opinion contraire qu'il attribue à un "philosophe très galant» très probablement Poullain de la Barre - pour qui «la nature n'a jamais dicté une telle loi» et qui voit dans cette domination une véritable "tyrannie», un privilège usurpé de manière déloyale par une éducation injuste.

Nous employons toutes sortes de moyens pour leur abattre leur courage; les forces seraient égales si l'éducation l'était aussi. (Lettre XXXVIII).

Ce discours tout à fait "éclairé» sur l'égalité naturelle des femmes et les raisons de la domination mâle dans la société n'aura cependant qu'une portée limitée. Si Rica montre de l'intérêt pour un tel discours, il ne peutencore yadhérer complètement; d'un point de vue philosophique, ce discours le séduit, mais il ne le fait pas sien. II l'écarte en affirmant que dans ce pays «l'on aime à soutenir des opinions extraordinaires et à réduire tout en paradoxe». Rica confirmera ensuite le patriarcat en faisant appel au Coran, qui proclame une réciprocité entre les époux tout en avantageant légèrement l'homme ${ }^{4}$. Cependant, malgré cette conclusion quelque peu régressive, le questionnement demeure et permet de révéler discrètement que, sur le plan rationnel, la subordination de la femme à l'homme n'est pas nécessairement conforme au code de la nature. La prééminence de l'homme sur la femme paraît alors avoir un fondement historique.

Les inégalités établies par une négation des capacités intellectuelles des femmes sont renforcées par la négation de leur capacité et de leur désir sexuels, comme en témoigne une

4. Voir à ce propos l'analyse de Pauline Kra, «Montesquieu and Women» dans French Women and the Age of Enlightenment éd. par Samia I. Spencer, Indiana University Press, Bloomington, 1984, p. 272-284. 
lettre adressée par Rica à Usbeck ${ }^{5}$. Montesquieu use du discours dans le discours et fait rapporter à Rica une histoire contée par une femme à ses amies. Nous voici donc devant un autre point de vue, celui d'une femme qui s'adresse à un public féminin. L'histoire racontée par Zulema et rapportée par Rica est celle d'Anaïs qui, maltraitée puis assassinée par son époux, est récompensée dans l'au-delà par un harem "d'hommes célestes destinés à ses plaisirs". Ce renversement de l'ordre terrestre semble exprimer l'idée de la perception par la femme de sa propre capacité sexuelle ${ }^{6}$. Ce conte dans le conte donne à imaginer un monde où la femme ne serait plus l'objet mais le sujet du désir et du plaisir.

La reconnaissance des mêmes désirs et capacités chez les femmes est d'une importance capitale car une telle implication mine le fondement moral du patriarcat basé sur l'idée de besoins différents. L'histoire d'Anaïs nous permet d'en juger. En effet, Anaìs envoie à ses compagnes terrestres un de ses célestes amants qui doit se faire passer pour leur époux Ibrahim et expulser celui-ci de son propre sérail. Ne se sentantnullement menacé par elles, le nouveau maître ne douta pas de ses femmes et ouvrit la porte du sérail à tous, organisant des fêtes où hommes et femmes se côtoyaient en toute liberté. Ce conte souligne que la jalousie des hommes ne vient pas du manque de vertu des femmes mais plutôt de la volonté des premiers de contrôler la sexualité de leurs conjointes.

Le discours de Rica semble souligner que l'ordre patriarcal dépendait de l'identification de l'autorité mâle avec la morale rationnelle et du contrôle de la sexualité féminine dans le but de sauvegarder l'héritage patrilinéaire.

De telles spéculations ne pourraient pas effleurer l'esprit d'Usbeck qui incarne les valeurs du patriarcat. À travers lui, Montesquieu analyse les fondements de ce système. Son attitude envers les femmes peut paraître ambigüe, dans la mesure où il établit d'emblée une différence entre les femmes

5. Lettre CXLI.

6. Kra, ibid., p. 276. 
en général et celles du sérail, celles dont il n'est pas le maître, et celles qui sont ses esclaves. De cette différenciation naît une contradiction, qui oppose le discours del' «apprenti philosophe» aux actes du maître de sérail. La différence entre elles est que les unes semblent intégrées à une humanité plus grande, celle des hommes, et que les autres en sont exclues par leur statut même d'esclaves.

En effet, lorsqu'Usbeck se fait le défenseur du divorce ${ }^{7}$ ou encore du suicide ${ }^{8}$, il défend des principes qu'il semble nier lorsque ses femmes sont concernées. C'est pour des raisons purement démographiques, dans le but d'expliquer «pourquoi la terre est moins peuplée" ${ }^{9}$, que Montesquieu aborde la question du divorce. Son discours est une approche théorique, presque philosophique, du problème du divorce :

II ne faut donc pas s'étonner si l'on voit chez les chrétiens tant de mariages fournir un si petit nombre de citoyens. Le divorce est aboli; les mariages mal assortis ne se raccommodent plus; les femmes ne passent plus, comme chez les Romains, successivement dans les mains de plusieurs maris, qui en tiraient, dans le chemin, le meilleur parti qu'il était possible.

C'est toujours du point de vue de la propagation de l'espèce qu'Usbeck s'interroge et critique le modèle de la claustration et de la distribution des femmes. Le danger de ce modèle réside entre autres dans les conséquences de l'excès de l'empire des sens : le danger de la perte d'énergie virile qui semble se mesurer ici en termes d'affaiblissement du futur de la lignée, garante du corps social. À la lettre CXIV, Usbeck dénonce le harem, où de nombreuses femmes enfermées sont gardées par un grand nombre d'eunuques. "Quelle perte, dit-il, pour la société que ce grand nombre d'hommes morts dès leur naissance! Quelle dépopulation ne doit-il pas s'en suivre! (...)

7. Notons que le divorce est permis en Islam, mais de manière favorable aux hommes uniquement, puisque les femmes sont en permanence sous la tutelle de l'homme (le père ou le mari).

8. cf. lettre LXXVI

9. Lettre CXIV. Voir les lettres CXIV à CXVI sur la question du divorce. 
Voilà comment un seul homme occupe à ses plaisirs tant de sujets de l'un et l'autre sexe, les faitmourir pour l'État, et les rend inutiles à la propagation de l'espèce." Ainsi se trouve exprimée la négation du corps anatomique au nom du corps de l'État. Les plaisirs du corps du sultan sont condamnés au nom du corps de l'État.

Cette notion de corps se retrouve aussi dans L'esprit des lois, où le corps anatomique se trouve différencié du corps social. La lecture du chapitre 4 du livre XXIII est particulièrement édifiante quant à la double acception du mot «famille» et de ses conséquences sur le dédoublement des corps des hommes et des femmes.

Dans un premier temps, le mot «famille» est pris dans le sens de corps social, signifiant d'abord la «famille du mari». Ce mot se trouve rapidement repris dans le sens de "propriété", et de cette partie de la famille "qui la perpétue». Le mot prend alors un sens anatomique et corporel, celui qui assure la propagation de l'espèce, à savoir le corps des femmes. Ce dédoublement du mot famille signale un autre dédoublement, celui de la notion de corps qui prend d'une part le sens de corps social décorporalisé, et d'autre part celui de corps réduit à sa corporalité : le corps féminin. Ce corps est alors «propriété» du corps social qui le contrôle et au service duquel il se trouve.

La pluralité et l'empire des femmes sont un danger en Perse, mais ils le sontégalementen France comme en témoigne la lettre CVII qui présente les conséquences néfastes d'une atmosphère excessivement féminisée : un roi et un royaume dirigés par les femmes. La France a été réduite à une situation où les femmes opèrent ensemble pour exercer l'autorité et allouer le pouvoir. "Lorsque j'arrivai en France, dit Usbeck, je trouvai le feu Roi absolument gouverné par les femmes (...) Ces femmes ont toutes des relations les unes avec les autres et forment une espèce de république dont les membres toujours actifs se secourent et se servent mutuellement: c'est comme un nouvel état dans l'État." Les femmes - corps anatomiques deviennent alors corps social microcosmique dans un corps social plus grand, celui de la nation. Ce nouveau corps, tel une 
tumeur, affaiblit le corps qu'il a investi, le pervertit; il en perturbe le bon fonctionnement. Les femmes sont perçues ici comme étant en dehors de leur place; elles brouillent les frontières, et là où les frontières deviennent floues, les hommes sont réduits à une position efféminée et le désordre s'ensuit nécessairement.

La perte d'énergie décrite comme sexuelle en Perse, l'est en terme d'argent en France : «Crois-tu Ibben qu'une femme s'avise d'être la maîtresse d'un ministre pour coucher avec lui. Quelle idée! C'est pour lui procurer cinq ou six placets tous les matins, et la bonté de leur naturel paraît dans l'empressement qu'elles ont à faire du bien à une infinité de gens malheureux qui leur procurent cent mille livres de rentes" (I. CVII). Cette relation qui lie l'argent et les femmes nous éclaire sur les résonnances introduites par la circulation des assignats dans le corps de la nation :

Tous coux qui étaient riches il y a encore six mois sont à présent dans la pauvreté, et coux qui n'avaient pas de pain regorgent de richesses. Jamais cos deux extrémités ne se sont touchées de si près. L'Étranger a toumé l'état comme un fripier foume un habit: il fait paraître dessus ce qui ótalt dessous; et ce qui b́tait dessus, il le met à l'envers. Quel désordre dans l'Étatl Quelle confusion dans les rangsl (I. CXXXVIII)

Ainsi, en investissant le corps de l'État, le corps étranger (femmes et Law) y sème le désordre et la confusion le mettant alors en péril; péril qui, dans la topologie du monde renversé, réside dans le fait que les maîtres (hommes ou nobles) vont devenir esclaves (femmes, laquais). Voilà qui sollicite l'interprétation sexuelle non seulement par le recours au fantasme du sérail où l'opposition maître-esclave se définit de façon sexuelle, mais par le recours à la métaphore de l'inversion (I.CXXXVIII). L'horreur de cet état retourné prend tout son sens, lu dans le contexte du discours médical de l'époque où la topographie de la différence sexuelle se trouve exprimée selon l'image du retournement. L'état monarchique «retourné» se trouve efféminé, dans le sens où les traités médicaux de l'époque décrivent le changement de sexe.

Cette métaphore du retournement inscrite dans Les lettres persanes renvoie à certains traités médicaux de l'époque qui, sans être cités directement, sont opératoires dans la réflexion 
de l'auteur' ${ }^{10}$. Thomas Laqueur rappelle qu'avant le XIXe siècle, le modèle de sexualité qui dominait la pensée anatomique présentait la femme non comme sexuellement différente de l'homme mais comme un homme inverti. Chacune des parties del'organe sexuel mâle avait, dans cette conception anatomique, son équivalent «retourné» dans les organes sexuels féminins ${ }^{11}$. À la lumière de cette conception de l'anatomie humaine, le texte littéraire prend une dimension nouvelle et permetà Marie-Claire Vallois de dire que l'assimilation du «mal dont souffre le corps monarchique à l'opération d'un retournement reviendrait, dans l'économie du texte, où le pouvoir sexuel se présente comme la métaphore du pouvoir social, à lire ce retournement comme une opération de transsexualité contre nature ${ }^{12}$. . Montesquieu se chargerait-il de mettre en place le système d'une autre opposition masculin-féminin dont la loi se trouverait dans une autre vision de la nature et du corps?

La lecture de L'esprit des lois et de la théorie des climats pourra sans doute nous éclairer. À travers la mise en place de l'appareil de la théorie des climats, Montesquieu en vient à justifier et à expliquer l'organisation sociale du point de vue sexuel $^{13}$. Le système climatique parvient à expliquer l'économie sexuelle qui semble devoir se traduire selon le problème de la distribution des femmes. Bourdieu montre comment, chez Montesquieu, la théorie de l'économie sexuelle, présentée comme la conclusion logique de l'observation scientifique se trouve en fait être l'inconscient qui motive la théorie des climats :

Lathéorie desclimatsesten effetun remarquableparadigme
de la mythologie «scientifique», discours fondé dans la
croyance (ou le préjugé) qui louche vers la science et qui
se caractérise donc par la coexistence de deux principes
entremélés de cohérence : une cohérence proclamée,

10. Je renvoie ici à Thomas Laqueur qui consacre une étude à l'histoire anatomique de la sexualité ( $\propto$ Amor Veneris, Vel Dulcedo Appelatur» dans Fragments for a History of the Human Body, Part 3, édité par Michel Feher avec Ramona Naddaff et Nadia Tazi [N.Y :Zone Books, 1989], pp.91-131).

11. Ibid., p. 94.

12. VALLOIR, Marie-Claire, «Rêverie orientale» et géopolitique du corps féminin chez Montesquieu, Romance Quarterly, vol. 38, n`3, août 1991.

13. Voir livre 16, chap.4. 
d'allure scientifique, qui s'affirme par la multiplication des signes extérieurs de la scientificité, et une cohérence cachée, mythique dans son principe ${ }^{14}$.

Bourdieu souligne que le type de discours où la pensée mythique du sexuel s'enclenche sur la théorie scientifique des climats est en fait le discours médical élaboré par John Arbuthnot qui, alliant la théorie des humeurs à la théorie des fibres, découvre l'imaginaire mythique d'une rêverie sur le corps, «l'air froid, dit Montesquieu, resserre les extrêmités des fibres extérieures de notre corps; cela augmente leur ressort, et favorise le retour du sang des extrémités vers le cœur. II diminue la longueur de ces mêmes fibres; il augmente donc par là leur force. L'air chaud, au contraire, relâche les extrémités des fibres, et les allonge; il diminue donc leur force et leur ressort" (livre XIV, chap.2).

Dans ce passage où les corps ne sont pas encore différenciés, sexualisés, se trouve déjà inscrit le principe d'une différenciation qui oppose d'une part le froid, le ressort, la force et d'autre part le chaud, le manque de ressort, de force. Dans la description physiologique, la polysémie des mots (ressort, puissance, équilibre...) révèle des oppositions gouvernées par l'opposition principielle du masculin et du féminin. La lecture du livre XVII, ch. 3 concernant le climat de l'Asie est très édifiante à ce propos. En effet, on apprend qu'en Asie - où «les lieux situés dans un climat très froid touchent immédiatement ceux qui sont dans un climat très chaud" - «les nations sont opposées aux nations du fort au faible; les peuples guerriers, braves et actifs touchent immédiatement des peuples efféminés, paresseux, timides : il faut donc que l'un soit conquis, et l'autre conquérant».

L'opposition du «maître» (de soi, donc des autres) et de "l'esclave» (des sens et du maître) oppose les hommes du Nord, «actifs, virils, tendus» à ceux du Midi condamnés à la passivité (féminine) par leur servitude à l'égard des sens; leur passion de la femme en est une qui énerve, affaiblit, amollit, 
prive de ressort et d'énergie : une passion qui somme toute «féminise».

En opposant l'Occident, le lieu du froid, à l'Orient, lieu du chaud, la théorie des climats féminise le corps de l'Orient pour lui opposer le corps «masculin» de l'Occident. Voilà donc que le climat gouverne la politique sexuelle et établit du même coup une idéologie des rôles sexuels très différenciés.

Cette idéologie de la différence (virilité/activité/ressort opposés à féminité/passivité/relâché), qui maintenants'impose selon le principe d'une loi naturelle (celle des températures), en vient, nous dit Bourdieu, à «faire disparaître l'histoire en réduisant le déterminisme historique (...) au déterminisme physique ${ }^{15}$ ".

Dans l'opposition masculin-féminin, proposée comme opposition du maître et de l'esclave, la réversibilité est toujours possible (je renvoie ici au harem et à sa structure réversible) et laisse anticiper un retournement du masculin en féminin, retournement rendu possible par le fait que dans ce modèle de dualité sexuelle, le masculin et le féminin relèvent du même corps. Le danger, pour Montesquieu, se trouve donc dans cette opposition maître-esclave à laquelle il propose l'alternative de l'opposition sexuelle «naturelle» que l'on trouve dans la société plus «naturelle» des Troglodytes.

En effet, dès le début du roman (I.XII), l'exemple des Bons Troglodytes établit un lien concret entre la croissance de la population et la vertu des pères qui consiste à "aimer leurs femmes", "élever leurs enfants" et par-dessus tout à "sentir que l'intérêt des particuliers se trouve toujours dans l'intérêt commun". Quant aux femmes troglodytes, leur vertu consiste à «rendre un Troglodyte heureux.» Le bonheur de la procréation est par ailleurs souligné dans le conte rapporté par Rica à la lettre CXXXXI. En outre, dans le chapitre 9 du livre VII de L'esprit des lois, Montesquieu semble favoriser un idéal féminin de chasteté, de simplicité et de retenue, qui éloigne les femmes de la sphère publique pour les situer dans la sphère domestique. 
L'opposition proposée n'est plus celle du maître et de l'esclave, mais plutôt celle du père et de la mère, du géniteur et de la génitrice. Ce partage représente les balbutiements d'une restructuration du paysage sexuel en ce sens qu'il semble renvoyer les hommes au corps de l'État, à la sphère publique politique et les femmes au corps de la génération, à la sphère privée et domestique. Voilà qui établit une différence qui n'est plus réversible, où la femme n'est plus l'envers de l'homme, mais son autre radical ${ }^{16}$.

Ce discours sera repris et articulé de manière plus radicale dans un projet plus vaste de domestication des femmes par les dirigeants de la Révolution qui ont réduit au silence les femmes publiques.

Nous conclurons simplement en soulignant que le contexte de l'articulation de cette idéologie de la différence était bel et bien politique, dans la mesure où les nouvelles luttes engagées étaient des luttes pour le pouvoir et la place à prendre dans la sphère publique.

Si à la Révolution française les frontières entre les classes ont été estompées, celles entre les hommes et les femmes ont été renforcées, rendues encore plus visibles. La création de la sphère publique bourgeoise a posé le problème de son occupation : qui des deux sexes devait légitimement occuper cette sphère? La biologie entra alors dans le discours et les différences sexuelles fondamentales nouvellement découvertes fournirent la matière nécessaire à l'établissement de nouvelles frontières.

\author{
Ilhame El Himdy, \\ Département d'Études françaises \\ Université de Montréal
}

16. BADINTER, Elizabeth, L'Un est L'Autre : des relations entre hommes et femmes. Paris, éditions Odile Jacob, 1986. 